\title{
Avaliação leucocitária de cães com linfoma submetidos ao protocolo de Madison-Wisconsin pela técnica convencional e citometria de fluxo
}

\section{Evaluation of leukocyte count in dogs with lymphoma submitted to the Madison-Wisconsin protocol by conventional technique and flow cytometry}

\author{
Letícia Abrahão Anai ${ }^{1 *}$; Flávia Eiras Dela Coleta ${ }^{2}$; Thiago Demarchi Munhoz²; \\ Andressa Francisca Silva Nogueira ${ }^{1}$; Lívia Maria Souza Semolin ${ }^{1}$; \\ Manuela Cristina Vieira ${ }^{1}$; Aureo Evangelista Santana ${ }^{3}$
}

\begin{abstract}
Resumo
O linfoma é o tumor de tecido hematopoético mais comum nos cães e um dos tumores malignos de maior ocorrência nesta espécie. É um ótimo modelo experimental para estudo devido a sua semelhança com o linfoma não-Hodgkin em humanos. Considerando a importância das alterações decorrentes da evolução desta neoplasia e aquelas ocorridas com o emprego da poliquimioterapia, avaliou-se o leucograma absoluto e a contagem de células $\mathrm{CD}^{4} 5^{+}$pela citometria de fluxo, no sangue de 25 cães com linfoma. Foram avaliados no momento do diagnóstico, uma vez por semana, durante as primeiras oito sessões quimioterápicas do protocolo de Madison-Wisconsin, e cujas contagens obtidas em contador automático convencional e por intermédio da citometria de fluxo, foram comparadas. Os resultados obtidos não revelaram diferenças estatisticamente significativas entre as duas técnicas utilizadas.
\end{abstract}

Palavras-chave: CD45, linfoma, cão

\begin{abstract}
Lymphoma is the most common hematopoietic tumor in dogs and one of the malignant tumors with higher occurrence in this species. It is a great experimental model due to its resemblance with the nonHodgkin lymphoma in humans. Considering the importance of the overall changes that result from to this kind of neoplastic tumor and those due to polichemotherapy this study aimed to evaluate the absolute leukon count and the total count of $\mathrm{CD}^{4} 5^{+}$cells in the blood of 25 dogs with lymphoma. Findings were crosschecked since diagnose and then once weekly during the first eight sessions of the Madison-Wisconsin chemotherapic protocol. Total granulocyte, lymphocyte and monocyte counts obtained from a conventional automatic counter and by flow cytometry were compared. Results did not reveal statistically significant changes between the two techniques.
\end{abstract}

Key words: CD45, lymphoma, dog

\footnotetext{
${ }^{1}$ Discente(s) de Doutorado do Curso em Medicina Veterinária, Universidade Estadual Paulista, Campus Jaboticabal, PR. E-mail: letanai@hotmail.com; dessafsn@yahoo.com.br; liviasemolin@yahoo.com.br; manu-vet@hotmail.com

${ }^{2}$ Drs. em Medicina Veterinária, Universidade Estadual Paulista, Campus Jaboticabal, PR. E-mail: flaviacoleta@uol.com.br; tdmunhoz@hotmail.com

${ }^{3}$ Prof. de Dept ${ }^{\mathrm{o}}$ de Medicina e Cirurgia Veterinária, Universidade Estadual Paulista, Campus Jaboticabal, PR. E-mail: santana@ fcva.unesp.br

* Autor para correspondência
} 


\section{Introdução}

O linfoma é uma neoplasia caracterizada por proliferação clonal de linfócitos malignos e pode originar-se na medula óssea, timo, baço, fígado, intestino, pele e linfonodos (VAIL; YOUNG, 2007; DALECK; CALAZANS; De NARDI, 2008). É a neoplasia hematopoiética que mais comumente afeta o cão (VAIL; OGILVIE, 1998), podendo representar de 7 a $9 \%$ das neoplasias malignas (MORRISON, 2005). Acomete principalmente animais de meiaidade ou idosos, que perfazem $80 \%$ dos casos (GREENLEE et al., 1990), não havendo predileção sexual (VAIL; OGILVIE, 1998). O tratamento de eleição para esta neoplasia é a quimioterapia antineoplásica, um dos protocolos mais utilizados é o de Madison-Wisconsin, uma combinação dos fármacos L-asparaginase, vincristina, prednisona, ciclofosfamida e doxorrubicina. Há relatos de que o referido protocolo é capaz de promover remissão completa em 92,3\% dos casos, com tempo de sobrevida de aproximadamente 397 dias para cães com linfoma (MORRISON, 2005). As etapas do tratamento quimioterápico do linfoma canino incluem indução da remissão, manutenção da remissão e reindução da remissão ou terapia de resgate (MACEWEN; YOUNG, 1996).

A mielotoxicidade é um fator limitante da quimioterapia antineoplásica, sendo a mais frequente e mais grave, por dois motivos. Primeiramente, porque pode comprometer a quimioterapia antineoplásica de maneira transitória ou definitiva, uma vez que pode implicar na diminuição da dose do quimioterápico e, por conseguinte, prejudicar a eficácia do tratamento. O segundo motivo está relacionado ao risco potencial de morte do paciente em decorrência de neutropenia séptica que pode se instalar devido a translocação de bactérias presentes na luz intestinal ou de foco infeccioso existente antes do início da quimioterapia antineoplásica (LANORE; DELPRAT, 2004).

A neutropenia é o evento mais frequente e mais grave dentre as citopenias decorrentes da quimioterapia antineoplásica. A maioria dos quimioterápicos tem ação mielotóxica, sendo que a ciclofosfamida na dose de 250 a $300 \mathrm{mg} / \mathrm{m}^{2}$ apresenta mielotoxicidade elevada e a vincristina, na dose de $0,75 \mathrm{mg} / \mathrm{m}^{2}$, moderada (LANORE; DELPRAT, 2004). A neutropenia geralmente persiste por três a cinco dias e, sendo que após a esse período a celularidade da medula óssea passa por uma fase de recuperação (LANORE; DELPRAT, 2004).

A citometria de fluxo é um recurso emergente na Medicina Veterinária que permite uma análise rápida, objetiva e quantitativa de células em suspensão (FALDYNA et al., 2001). O CD45 é o antígeno comum aos leucócitos e é expresso em intensidades diferentes na superfície de todos os leucócitos (GRINDEM, 1996). De acordo com Weiss (2004) o CD45 pode ser útil na avaliação de leucemias granulocíticas agudas e linfomas. Além disso, quando consideradas as variáveis morfológicas com base na complexidade interna, o anticorpo monocolonal correspondente é, usualmente, utilizado para a confecção de "gates" para diferenciar os leucócitos de eritrócitos e debris celulares, sendo possível a realização de um mielograma por intermédio da citometria de fluxo, de tal forma a separar cada linhagem, permitindo que seu perfil de expressão antigênica seja estudado (COMAZZI et al., 2006).

Neste ensaio, objetivou-se avaliar o leucograma e a contagem de células $\mathrm{CD}^{4} 5^{+}$, no sangue periférico de cães com linfomas, no momento do diagnóstico e durante as oitos primeiras sessões quimioterápicas do protocolo Madison-Wisconsin. Comparando assim o método convencional (contador automático) e a citometria de fluxo.

\section{Material e Métodos}

O grupo tratado incluiu vinte e cinco (25) cães portadores de linfoma, atendidos pelo Serviço de Oncologia Veterinária do Hospital Veterinário "Governador Laudo Natel" da FCAVUNESP. O diagnóstico de linfoma foi firmado a 
partir dos achados clínicos, citopatológicos e/ou histopatológicos de tecido linfóide, estes últimos de acordo com critérios de classificação morfológica descritos por Fournel-Fleury et al. (2002). Dos 25 animais que compuseram o ensaio em tela, quatorze eram machos $(56 \%)$ e onze eram fêmeas $(44 \%)$. A maioria dos cães eram da raça Rottweiler (28\%), seguida por cães sem raça definida - SRD (20\%), Boxers (16\%), Poodles (8\%), Cocker Spaniel (8\%), Husky Siberiano (4\%), Teckel (4\%), Labrador (4\%), American Stadfordshire Terrier (4\%) e Golden Retriever (4\%). A idade variou de três a 15 anos $(7,4$ $\pm 2,8)$ e o peso de 6,9 e $49 \mathrm{Kg}(27,4 \pm 11,5)$.

Os animais com linfoma foram submetidos ao protocolo quimioterápico de Madison-Wisconsin, que incluiu sulfato de vincristina $\left(0,75 \mathrm{mg} / \mathrm{m}^{2}, \mathrm{IV}\right)$, L-asparaginase (400 UI/kg, IM), ciclofosfamida (250 mg/m $\mathrm{m}^{2}$, VO), doxorrubicina $\left(30 \mathrm{mg} / \mathrm{m}^{2}\right)$ e prednisona $(2-0,5 \mathrm{mg} / \mathrm{kg} / \mathrm{dia})$.
As amostras de sangue destinadas à obtenção do leucograma e quantificação de células $\mathrm{CD} 45^{+}$, foram coletadas por venopunção da jugular e envasadas em tubos a vácuo, contendo EDTA dipotássico $\left(\mathrm{K}_{2}-\right.$ EDTA). As contagens globais dos leucócitos foram obtidas com auxílio de um contador automático de células (ABC Vet HORIBA ABX - São Paulo/ Brasil) e as contagens diferenciais dos leucócitos por intermédio de esfregaços sanguíneos corados com uma mistura de Metanol, May-Grunwald e Giemsa - MGG.

Foram coletadas nove amostras de sangue, sendo a primeira no momento do diagnóstico e, as demais, uma semana após cada sessão de quimioterápica antineoplásica. Como valores de referência utilizaram-se aqueles estabelecidos e validados junto ao "Laboratório de Patologia Clínica Veterinária Prof. Dr. Joaquim Martins Ferreira Neto", do Hospital Veterinário Governador Laudo Natel, FCAV/Unesp, Campus de Jaboticabal, descrito na tabela 1 .

Tabela 1. Valores de referência para leucograma de cães, estabelecidos e validados junto ao Laboratório de Patologia Clínica Veterinária Prof. Dr. Joaquim Martins Ferreira Neto do Hospital Veterinário "Governador Laudo Natel" da Faculdade de Ciências Agrárias e Veterinárias. Jaboticabal, 2011.

\begin{tabular}{lcc}
\hline Parâmetros & Valores & $\%$ \\
\hline Leucócitos Totais & $6-18 / \mathrm{uL}$ & - \\
Neutrófilos & $3,6-13,8$ & $60-77$ \\
Bastonetes & $0-0,5$ & $0-3$ \\
Eosinófilos & $1,2-1,8$ & $2-10$ \\
Linfócitos & $0,72-5,4$ & $13-30$ \\
Monócitos & $0,18-1,8$ & $3-10$ \\
\hline
\end{tabular}

Fonte: Schalm's Veterinary Hematolgy, 5th Edition, 2000.

As amostras de sangue destinadas à análise citométrica foram processadas logo após a coleta, no Laboratório de Patologia Clínica Veterinária, precendentemente referido, utilizando-se de três tubos Falcon, de acordo com a sequência descrita na tabela 2 .

Após homogeneização do sangue, os tubos foram incubados no escuro e em temperatura ambiente, por 20 minutos. Um mililitro de tampão de lise de hemácias (FACS Lysing Solution Becton Dickinson) foi adicionado em cada tubo e, novamente, as amostras foram homogeneizadas e incubadas por mais dez minutos, à temperatura ambiente, no escuro. Em seguida, foi realizada a lavagem do material com solução salina tamponada com fosfato $0,01 \mathrm{M}$, pH entre 7,4 e 7,6 (PBS) por 
três vezes, adicionando-se dois mililitros de PBS por lavagem. Após as lavagens, adicionaram-se 0,5 $\mathrm{mL}$ de PBS a 1\% de formol, para a preservação das células, até o momento da leitura.

As amostras foram analisadas em até três dias, após o preparo, com o auxílio de um citômetro de fluxo FACSCANTO (Becton Dickinson, San Jose, CA), alocado junto ao Laboratório de Micologia, do Departamento de Análises Clínicas, da Faculdade de Ciências Farmacêuticas de Araraquara - Unesp. Foram obtidos 10000 eventos, para classificação e quantificação de células $\mathrm{CD}^{+} 5^{+}$, utilizandose do programa FACSDiva (BD) (Figura 1). Os valores absolutos para as células $\mathrm{CD} 45^{+}$sanguíneas foram calculados com base nos valores relativos, fornecidos pelo programa, e contagens de leucócitos totais obtidas no leucograma.

A análise estatística seguiu a Análise de Variância do sistema SAS, cujos valores médios obtidos para as diferentes características estudadas foram comparados pelo Teste de Tukey com um nível de probabilidade igual a 5\% (SNEDECOR; COCHRAN, 1987).

Tabela 2. Preparo das amostras de sangue periférico de cães linfomatosos, com vistas à análise citométrica. Jaboticabal, 2011.

\begin{tabular}{lllllll}
\hline Tubo & Amostra & Volume Anticorpo & Molécula & Fluorocromo & Anticorpo Monoclonal & Tipo celular \\
\hline 1 & $100 \mu \mathrm{L}$ & - & - & - & - & - \\
2 & $100 \mu \mathrm{L}$ & $2 \mu \mathrm{L}$ & $\gamma 2$ & FITC & MCA1212 & Isotipo controle \\
3 & $100 \mu \mathrm{L}$ & $2 \mu \mathrm{L}$ & CD45 & FITC & MCA1042F & Leucócitos \\
\hline
\end{tabular}

Fonte: Elaboração dos autores.

Figura 1. Distribuição das populações leucocitárias observadas à citometria de fluxo, de acordo com o tamanho e granularidade das células, superpostas à população de células $\mathrm{CD} 45^{+}$no sangue de cão com linfoma. Jaboticabal, 2011.

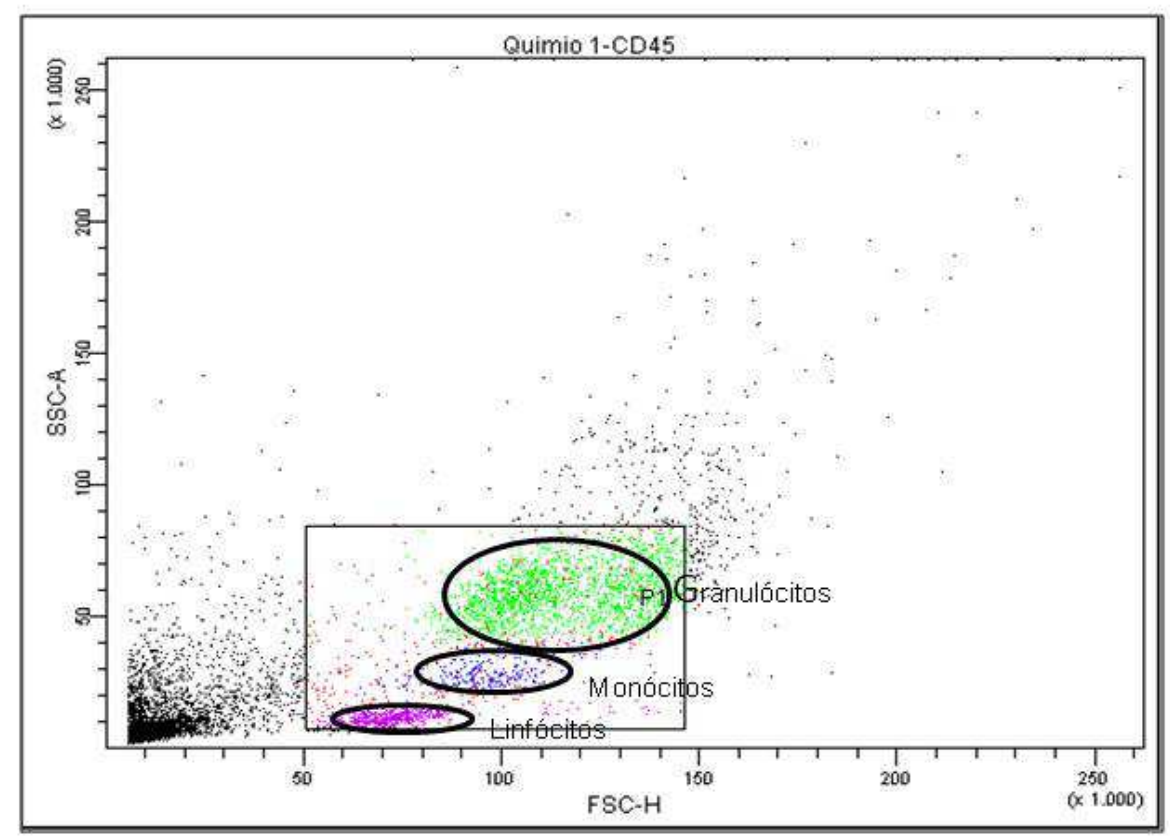

Fonte: Elaboração dos autores. 


\section{Resultados e Discussão}

$\mathrm{Na}$ comparação das técnicas de contagens globais de neutrófilos, linfócitos e monócitos, por intermédio da citometria de fluxo (imunomarcagem de $\mathrm{CD}^{4} 5^{+}$) e contagem automatizada (contador automático ABC-VET), foi verificado que os resultados obtidos foram bastante semelhantes em ambas as técnicas. Podendo ser verificado nas tabelas 3 e 4 .

Tabela 3. Valores médios obtidos para as contagens absolutas de granulócitos, linfócitos e monócitos, por intermédio da citometria de fluxo, de 25 cães com linfoma no momento do diagnóstico e uma semana após cada sessão de quimioterapia antineopláscia com o protocolo Madison-Wiscosin. Jaboticabal, 2011.

\begin{tabular}{|c|c|c|c|c|c|c|c|c|c|c|}
\hline & Diag & $\mathbf{V}+\mathbf{L}-\mathbf{a}+\mathbf{P}$ & $\mathbf{C}+\mathbf{P}$ & $\mathbf{V}+\mathbf{P}$ & $\mathbf{D}+\mathbf{P}$ & $\mathbf{V}$ & C & $\mathbf{V}$ & D & $\mathbf{P}$ \\
\hline $\begin{array}{l}\text { Neutrófilos } \\
\left(\mathbf{x 1 0}^{3} / \mathbf{u L}\right)\end{array}$ & $11,42 \mathrm{a}$ & $6,71 b$ & $8,27 \mathrm{~b}$ & $4,87 \mathrm{~b}$ & $5,71 b$ & $4,47 b$ & $5,27 \mathrm{~b}$ & $4,27 \mathrm{~b}$ & $5,55 \mathrm{~b}$ & $<0,05$ \\
\hline $\begin{array}{l}\text { Linfócitos } \\
\left(\times 10^{3} / \mathrm{uL}\right)\end{array}$ & $2,87 \mathrm{a}$ & $1,77 b$ & $1,06 \mathrm{~b}$ & $1,13 b$ & $0,91 b$ & $1,26 \mathrm{~b}$ & $0,81 b$ & $1,07 \mathrm{~b}$ & $1,22 b$ & $<0,05$ \\
\hline $\begin{array}{l}\text { Monócitos } \\
\left(\times 10^{3} / \mathbf{u L}\right)\end{array}$ & $0,35 \mathrm{a}$ & $0,30 \mathrm{a}$ & $0,21 \mathrm{a}$ & $0,13 \mathrm{a}$ & $0,12 \mathrm{a}$ & $0,13 \mathrm{a}$ & $0,05 \mathrm{a}$ & $0,17 \mathrm{a}$ & $0,16 \mathrm{a}$ & 0,10 \\
\hline
\end{tabular}

Diag: diagnóstico; V: vincristina; L-a: L-asparaginase; P: prednisona; C: ciclofosfamida; D: doxorrubicina. $\mathrm{p}<0,05$ letras iguais na mesma linha não diferem entre si.

Fonte: Elaboração dos autores.

Tabela 4. Valores médios obtidos para o leucograma de 25 cães portadores de linfoma no momento do diagnóstico e uma semana após cada sessão quimioterápica do protocolo Madison-Wiscosin. Jaboticabal, 2011.

\begin{tabular}{lcccccccccc}
\hline & Diag & $\mathbf{V}+\mathbf{L}-\mathbf{a}+\mathbf{P}$ & $\mathbf{C}+\mathbf{P}$ & $\mathbf{V}+\mathbf{P}$ & $\mathbf{D}+\mathbf{P}$ & $\mathbf{V}$ & $\mathbf{C}$ & $\mathbf{V}$ & $\mathbf{D}$ & $\mathbf{p}$ \\
\hline $\begin{array}{l}\text { Neutrófilos } \\
\mathbf{( x 1 0} / \mathbf{u L})\end{array}$ & $11,09 \mathrm{a}$ & $7,02 \mathrm{~b}$ & $8,55 \mathrm{~b}$ & $5,23 \mathrm{~b}$ & $6,13 \mathrm{~b}$ & $4,66 \mathrm{~b}$ & $8,06 \mathrm{~b}$ & $4,70 \mathrm{~b}$ & $5,21 \mathrm{~b}$ & $<0,05$ \\
$\begin{array}{l}\text { Linfócitos } \\
(\mathbf{x 1 0} / \mathbf{u L})\end{array}$ & $2,47 \mathrm{a}$ & $0,92 \mathrm{~b}$ & $0,84 \mathrm{~b}$ & $0,95 \mathrm{~b}$ & $1,00 \mathrm{~b}$ & $1,25 \mathrm{~b}$ & $1,02 \mathrm{~b}$ & $1,27 \mathrm{~b}$ & $0,94 \mathrm{~b}$ & $<0,05$ \\
$\begin{array}{l}\text { Monócitos } \\
(\mathbf{x 1 0} / \mathbf{u L})\end{array}$ & $0,69 \mathrm{a}$ & $0,63 \mathrm{a}$ & $0,60 \mathrm{a}$ & $0,41 \mathrm{a}$ & $0,38 \mathrm{a}$ & $0,37 \mathrm{a}$ & $0,32 \mathrm{a}$ & $0,29 \mathrm{a}$ & $0,23 \mathrm{a}$ & 0,10 \\
\hline
\end{tabular}

Diag: diagnóstico; V: vincristina; L-a: L-asparaginase; P: prednisona; C: ciclofosfamida; D: doxorrubicina. $\mathrm{p}<0,05$ letras iguais na mesma linha não diferem entre si.

Fonte: Elaboração dos autores.

As contagens globais obtidas para neutrófilos, linfócitos e monócitos, por intermédio da citometria de fluxo, foram comparadas àquelas obtidas pelo método automatizado (Contador de Células ABCVET), cujos resultados estão apresentados na tabela 5. O valor médio relativo obtido para os neutrófilos foi de $72,54 \%$ pela citometria de fluxo e $75,16 \%$ pelo método automatizado. Para os linfócitos o valor médio relativo foi de $15,78 \%$ por citometria de fluxo e 13,04\% na contagem automatizada e, no caso dos monócitos, o valor médio relativo obtido na citometria foi de $2,15 \%$ e na contagem automatizada igual a $4,91 \%$. 
Tabela 5. Valores médios relativos (\%) obtidos para granulócitos, linfócitos e monócitos, com o uso de contador automático e por citometria de fluxo, no sangue de 25 cães com linfoma, durante o protocolo quimioterápico M-W, Jaboticabal, 2011.

\begin{tabular}{cccc}
\hline & Neutrófilos & Linfócitos & Monócitos \\
\hline Contador Automático & $75,16 \pm 5,46$ & $13,04 \pm 2,83$ & $4,91 \pm 1,27$ \\
C.F & $72,54 \pm 4,78$ & $15,78 \pm 3,4$ & $2,15 \pm 0,68$ \\
\hline P & 0,15 & 0,23 & $<0,0001$ \\
\hline
\end{tabular}

C.F: citometria de fluxo. Valores de $\mathrm{p}<0,05$ significativo a $5 \%$.

Fonte: Elaboração dos autores.

Quando comparadas as duas técnicas utilizadas (citometria de fluxo e o método automatizado), verificou-se que houve diferenças significativas apenas entre os monócitos, sendo que as contagens de neutrófilos e linfócitos não apresentaram diferenças significativas em função das técnicas de contagem empregadas. Tais diferenças entre as contagens monócitárias, na comparação das duas técnicas, talvez, possam ser atribuídas à pequena quantidade destas células no sangue periférico, como também em razão da sua grande variabilidade numérica. Weiss (2004) correlacionou mielogramas obtidos de forma convencional àqueles obtidos por citometria (imunofenotipagem de $\mathrm{CD} 45^{+}$), em amostras de medula óssea de cães com desordens hematológicas, e obteve coeficiente de correlação igual a 0,90 entre neutrófilos e linfócitos, asseverou boa associação entre as duas técnicas, ainda que no caso dos monócitos, o índice de correlação entre as referidas técnicas tenha sido baixo $(0,54)$.

Com relação a análise do leucograma durante as sessões, podemos observar que a média da contagem absoluta dos neutrófilos apresenta uma diferença significativa apenas no momento do diagnóstico em relação aos demais momentos. O aumento da quantidade de neutrófilos é um achado encontrado em 25 a 40\% dos cães portadores de linfoma (VAIL; YOUNG, 2007). Neste estudo, o encontro desse aumento, provavelmente refletiu uma condição inflamatória decorrente do tumor (SCHULTZE, 2000).

De outra parte, a presença da redução da média de neutrófilos foi observada principalmente após a quimioterapia com a vincristina, achado que não foi relatado nos trabalhos de Tomiyasu et. al. (2010), que encontraram uma baixa porcentagem de animais com neutropenia. Sendo assim, e nas condições do ensaio em questão, pode-se considerar que a vincristina foi capaz de promover uma maior supressão de leucócitos circulantes em função da diminuição de neutrófilos segmentados, quando comparada aos demais quimioterápicos utilizados.

A ciclofosfamida, apesar de levar a uma maior mielossupressão, principalmente traduzida por neutropenia (BARGER; GRINDEM, 2000), não revelou, ao menos neste ensaio, tal intensidade de ação mielossupressora, contrariando, os achados de Tomiyasu et. al. (2010) que observaram a media de $20,6 \%$ de cães com mielossupressão. É bem possível que tal discrepância tenha sido devida à via de administração do quimioterápico, pois neste estudo foi utilizada a via oral e, no daqueles, a intravenosa.

Já, com relação à contagem absoluta dos linfócitos observou-se diferença significativa apenas no momento do diagnóstico, no qual $12 \%$ dos cães apresentaram linfocitose. Após as primeiras sessões quimioterápicas, mais de $50 \%$ dos animais apresentaram-se linfopênicos, fato que atesta a acentuada toxicidade dos quimioterápicos no tecido linfóide, tal qual asseverado por Gauthier et al. (2005). Ainda com relação aos leucócitos mononucleares observou-se diminuição progressiva no número absoluto de monócitos, mas os valores médios encontrados se mantiveram dentro dos valores de referência, para a espécie canina, e também não se observaram diferenças significativas entre os momentos. 


\section{Conclusão}

Os resultados obtidos no presente estudo permitem concluir que o leucograma de cães com linfoma pode ser avaliado tanto por intermédio de contadores eletrônicos como por meio da citometria de fluxo. E as principais alterações no leucograma foram observadas no momento do diagnóstico e, portanto, se referem ao linfoma; e a maior mielosupressão leucocitária é imposta pela vincristina.

\section{Agradecimentos}

Os autores agradecem a Fundação de Amparo à Pesquisa do Estado de São Paulo (Fapesp) pelo apoio financeiro.

\section{Comitê de Ética e Biossegurança}

Este estudo foi aprovado pela Comissão de Ética e Bem-estar Animal da FCAV - UNESP (protocolo $n^{\circ}$ 007285-09).

\section{Referências}

BARGER, A. M.; GRINDEN, C. B. Hematologic abnormalities associated with cancer therapy. In: FELDMAN, B. F.; ZINKL, J. G.; JAIN, N. C. Schalm's veterinary hematology. 5. ed. Toronto: Lippincott Williams Wilkins, 2000. p. 676-681.

COMAZZI, S.; GELAIN, M. E.; SPAGNOLO, V.; RONDATO, F.; GUGLIELMINO, R.; SARTORELLI, P. Flow cytometric patterns in blood from dogs with non-neoplasic and neoplasic hematologic diseases using double labeling for CD18 and CD45. Veterinary Clinical Pathology, Santa Bárbara, v. 35, n. 1, p. 47-54, 2006. Disponível em: <http://onlinelibrary.wiley.com/ doi/10.1111/j.1939-165X.2006.tb00088.x/pdf>. Acesso em: 12 abr. 2012.

DALECK, C. R.; CALAZANS, S. G.; De NARDI, A. B. Linfomas. In: DALECK, C. R.; De NARDI, A. B.; RODASKI, S. Oncologia em cães e gatos. São Paulo: Roca, 2008. p. 481-505.
FALDYNA, M.; LEVÁ, L.; KNÖTIGOVÁ, P.; TOMAN, M. Lymphocyte subsets in peripheral blood of dogs - a flow cytometric study. Veterinary Immunology and Immunopathology, Netherlands, v. 82, n. 1-2, p. 23-37, 2001. Disponível em: <http://www.sciencedirect.com/ science/article/pii/S0165242701003373>. Acesso em: 29 jan. 2012.

FOURNEL-FLEURY, C.; PONCE, F.; FELMAN, P.; BLAVIEI, A.; BONNEFONT, C.; CHABANNE, L.; MARCHAL, T.; CADORE, J. L.; GOY-THOLLOT, I.; LEDIEW, D.; GHERNATI, I.; MAGNOL, J. P. Canine T-cell lymphomas: a morphological, immunological, and clinical study of 46 new cases. Veterinary Pathology, Cary, v. 39, n. 1, p. 92-109, 2002.

GAUTHIER, M. J.; AUBERT, I.; ABRAMS-OGG, A.; WOODS, J. P.; BIENZLE, D. The Immunophenotype of peripheral blood lymphocytes in clinically healthy dogs and dogs with lymphoma in remission. Journal of Veterinary Internal Medicine, Lawrence, v. 19, n. 2, p. 193-199, 2005.

GREENLEE, P. G.; FILIPPA, D. A.; QUIMBY, F. W.; PATNAIK, A. K.; CALVANO, S. E.; MATUS, R. E.; KIMMEL, M.; HURVITZ, A. I.; LIEBERMAN, P. H. Lymphomas in dogs: a morphologic, immunologic, and clinical study. Cancer, Philadephia, v. 66, p. 480-490, 1990. Disponível em: <http://onlinelibrary.wiley.com/ doi/10.1002/1097-0142(19900801)66:3\%3C480::AIDCNCR2820660314\%3E3.0.CO;2-X/pdf >. Acesso em: 15 dez. 2011.

GRINDEM, C. B. Blood cell markers. The Veterinary Clinics of North America: Small Animal Practice, Philadelphia, v. 26, n. 5, p. 1043-1065, 1996.

LANORE, D.; DELPRAT, C. Quimioterapia anticancerigena. São Paulo: Roca, 2004. 191 p.

MACEWEN, E. G.; YOUNG, K. M. Canine lymphoma and lymphoid leukemias. In: WITHROW, S. J.; MACEWEN, E. G. Small animal clinical oncology. 2. ed. Philadelphia: W. B. Saunders Company, 1996. cap. 28 B, p. 451-479.

MORRISON, W .B. Lymphoma in dogs and cats. Texas: Teton New Media, 2005. 124 p.

SCHULTZE, A. E. Interpretation of canine leukocyte responses. In: FELDMAN, B. F.; ZINKL, J. G.; JAIN, N. C. Schalm's veterinary hematology. 5. ed. Baltimore: Lippincott Williams \& Wilkins, 2000. p. 366-381.

SNEDECOR, G. W.; COCHRAN, W. G. Statistical methods. 6. ed. Ames: Iowa State University, 1987. 593 p. 
TOMIYASU, H.; TAKAHASHI, M.; FUJINO, Y.; OHNO, K.; TSUJIMOTO, H. Gastrointestinal and hematologic adverse events after administration of vincristina, cyclophosphamide, and doxorrubicin in dogs with lymphoma that underwent a combination multidrug chemotherapy protocol. The journal of veterinary medical science, Tokyo, v. 72, n. 11, p. 1391-1397, 2010. Disponível em: <https:/www.jstage.jst.go.jp/article/ jvms/72/11/72_10-0176/_article $>$. Acesso em: 10 nov. 2011.

VAIL, D. M.; OGILVIE, G. K. Neoplasias linfóides. In: BICHARD, S. J.; SHERDING, R. G. Manual Saunders - clínica de pequenos animais. São Paulo: Roca, 1998. cap. 6 , p. 218-225.
VAIL, D. M.; YOUNG, K. M. Hematopoietic tumors. In: WITHROW, S. J.; VAIL, D. M. Small animal clinical oncology. 4. ed. Missouri: Saunders, 2007. p. 699-784.

WEISS, D. J. Flow cytometric evaluation of canine bone marrow based on intracytoplasmic complexity and CD45 expression. Veterinary Clinical Pathology Journal, Boston, v. 33, n. 2, p. 96-101, 2004. Disponível em: <http://onlinelibrary.wiley.com/doi/10.1111/j.1939165X.2004.tb00355.x/pdf>. Acesso em: 10 nov. 2011. 